The Journal of $\mathbf{N}_{\text {onlinear }} \mathbf{S}$ ciences and Applications

http://www.tjnsa.com

\title{
POSITIVE SOLUTIONS FOR BOUNDARY VALUE PROBLEM OF NONLINEAR FRACTIONAL DIFFERENTIAL EQUATION
}

\author{
TINGTING QIU $^{1 *}$ AND ZHANBING BAI ${ }^{2}$
}

\begin{abstract}
We investigate the positive solution of nonlinear fractional differential equation with semi-positive nonlinearity

$$
\left\{\begin{array}{l}
D_{0^{+}}^{\alpha} u(t)+f(t, u(t))=0, \quad 0<t<1 \\
u(0)=u^{\prime}(1)=u^{\prime \prime}(0)=0
\end{array}\right.
$$

where $2<\alpha \leq 3$ is a real number, $D_{0^{+}}^{\alpha}$ is the Caputo's differentiation, and $f:[0,1] \times[0, \infty) \rightarrow(-\infty, \infty)$. By use of Krasnosel'skii fixed point theorem, the existence results of positive solution are obtained.
\end{abstract}

\section{INTRODUCTION AND PRELIMINARIES}

Fractional differential equations have been of great interest recently. It is caused both by the intensive development of the theory of fractional calculus itself and by the applications of such constructions in various sciences such as physics, mechanics, chemistry, engineering, etc. For details, see [4, 6, 7, 8] and the references therein.

It should be noted that most of papers and books on fractional calculus are devoted to the solvability of linear initial fractional differential equations in terms of special functions [9]. Recently, there are some papers deal with the existence and multiplicity of solution (or positive solution) of nonlinear initial fractional differential equation by the use of techniques of nonlinear analysis (fixed-point theorems, Leray-Shauder theory, etc.), see [2, 3, 5, 11, 12].

However, there are few papers consider the semipositone nonlinearity differential equations of fractional order. No contributions exist, as far as we know,

Date: Received: 4 September 2008; Revised: 28 September 2008.

* Corresponding author.

2000 Mathematics Subject Classification. Primary 34B15; Secondary 34B18.

Key words and phrases. Fractional differential equation; Positive solution; Fixed-point theorem. 
concerning the positive solution with semipositone nonlinearity of the following problem:

$$
\left\{\begin{array}{l}
D_{0^{+}}^{\alpha} u(t)+f(t, u(t))=0, \quad 0<t<1, \\
u(0)=u^{\prime}(1)=u^{\prime \prime}(0)=0
\end{array}\right.
$$

where $2<\alpha \leq 3$ is a real number, $D_{0^{+}}^{\alpha}$ is the Caputo's differentiation, and $f:[0,1] \times[0, \infty) \rightarrow(-\infty, \infty)$ is continuous.

In this paper, we firstly derive the corresponding Green's function, then we give the properties of Green's function,finally,the existence of positive solution with semipositone nonlinearity are obtained by Krasnosel'skii fixed point theorem.

Here, a positive solution $u^{*}$ of (1.1) will mean a solution $u^{*}$ of (1.1) satisfying $u^{*}(t)>0,0<t<1$.

For the convenience of the reader, we present here the necessary definitions from fractional calculus theory. These definitions can be found in the recent literature.

Definition 1.1. The Riemann-Liouville fractional integral of order $\alpha>0$ of a function $f:[0,1] \rightarrow R$ is given by

$$
I_{0+}^{\alpha} f(t)=\frac{1}{\Gamma(\alpha)} \int_{0}^{t}(t-s)^{\alpha-1} f(s) d s
$$

provided that the right side is pointwise defined on $(0, \infty)$.

Definition 1.2. The Caputo's fractional derivative of order $\alpha>0$ of a function $f \in A C^{n}[0,1]$ is given by

$$
D_{0^{+}}^{\alpha} f(t)=\frac{1}{\Gamma(n-\alpha)} \int_{0}^{t} \frac{f^{(n)}(s)}{(t-s)^{\alpha-n+1}} d s
$$

where $n-1<\alpha \leq n$, provided that the right side is pointwise defined on $(0, \infty)$.

From the definition we can obtain the following lemma:

Lemma 1.3. Assume that $u \in C^{n}[0,1]$, then

$$
I_{0+}^{\alpha} D_{0+}^{\alpha} u(t)=u(t)-C_{1}-C_{2} t-\cdots-C_{n} t^{n-1}
$$

where $C_{i} \in R, \quad i=1,2, \ldots, n, n=[\alpha]+1$.

Lemma 1.4. 6] The relation

$$
I_{a+}^{\alpha} I_{a+}^{\beta} \varphi=I_{a+}^{\alpha+\beta} \varphi
$$

is valid in following case

$$
\operatorname{Re} \beta>0, \operatorname{Re}(\alpha+\beta)>0, \varphi(x) \in L_{1}(a, b) .
$$

Lemma 1.5. Given $f \in C[0,1]$, and $2<\alpha \leq 3$, the unique solution of

$$
\begin{aligned}
& D_{0^{+}}^{\alpha} u(t)+f(t)=0, \quad 0<t<1, \\
& u(0)=u^{\prime}(1)=u^{\prime \prime}(0)=0 .
\end{aligned}
$$

is

$$
u(t)=\int_{0}^{1} G(t, s) f(s) d s
$$


where

$$
G(t, s)= \begin{cases}\frac{(\alpha-1) t(1-s)^{\alpha-2}-(t-s)^{\alpha-1}}{\Gamma(\alpha)}, & 0 \leq s \leq t \leq 1, \\ \frac{t(1-s)^{\alpha-2}}{\Gamma(\alpha-1)}, & 0 \leq t \leq s \leq 1 .\end{cases}
$$

Proof. We may apply Lemma1.3 to reduce Eq.(1.2) to an equivalent integral equation

$$
u(t)=-I_{0+}^{\alpha} f(t)+C_{1}+C_{2} t+C_{3} t^{2}
$$

for some $C_{i} \in R, \quad i=1,2,3$. By Lemma1.4 we have

$$
\begin{gathered}
u^{\prime}(t)=-D_{0+}^{1} I_{0+}^{\alpha} f(t)+C_{2}+2 C_{3} t=-D_{0+}^{1} I_{0+}^{1} I_{0+}^{\alpha-1} f(t)+C_{2}+2 C_{3} t \\
=-I_{0+}^{\alpha-1} f(t)+C_{2}+2 C_{3} t \\
u^{\prime \prime}(t)=-D_{0+}^{1} I_{0+}^{\alpha-1} f(t)+2 C_{3}=-D_{0+}^{1} I_{0+}^{1} I_{0+}^{\alpha-2} f(t)+2 C_{3}=-I_{0+}^{\alpha-2} f(t)+2 C_{3} .
\end{gathered}
$$

From $u(0)=u^{\prime}(1)=u^{\prime \prime}(0)=0$, one has

$$
C_{1}=0, C_{2}=\frac{1}{\Gamma(\alpha-1)} \int_{0}^{1}(1-s)^{\alpha-2} f(s) d s, C_{3}=0 .
$$

Therefore, the unique solution of problem (1.2) is

$$
\begin{aligned}
u(t) & =-\frac{1}{\Gamma(\alpha)} \int_{0}^{t}(t-s)^{\alpha-1} f(s) d s+\frac{1}{\Gamma(\alpha-1)} \int_{0}^{1} t(1-s)^{\alpha-2} f(s) d s \\
& =\int_{0}^{t}\left[\frac{t(1-s)^{\alpha-2}}{\Gamma(\alpha-1)}-\frac{(t-s)^{\alpha-1}}{\Gamma(\alpha)}\right] f(s) d s+\int_{t}^{1} \frac{t(1-s)^{\alpha-2}}{\Gamma(\alpha-1)} f(s) d s \\
& =\int_{0}^{1} G(t, s) f(s) d s
\end{aligned}
$$

The proof is complete.

Lemma 1.6. The function $G(t, s)$ defined by Eq. (1.3) satisfies

(1) $G(t, s)>0$, for $t, s \in(0,1)$;

(2)

$$
\min _{1 / 4 \leq t \leq 3 / 4} G(t, s) \geq \frac{1}{4} \max _{0 \leq t \leq 1} G(t, s)=\frac{1}{4} G(1, s), \quad \text { for } 0<s<1 .
$$

Proof. Setting

$$
g_{1}(t, s)=\frac{(\alpha-1) t(1-s)^{\alpha-2}-(t-s)^{\alpha-1}}{\Gamma(\alpha)}, g_{2}(t, s)=\frac{t(1-s)^{\alpha-2}}{\Gamma(\alpha-1)} .
$$

(1) For $g_{1}(t, s)$, since $2<\alpha \leq 3,0 \leq s \leq t \leq 1$ we can obtain

$$
(\alpha-1) t(1-s)^{\alpha-2} \geq t(1-s)^{\alpha-2} \geq t(t-s)^{\alpha-2} \geq(t-s)^{\alpha-1}
$$

we get $g_{1}(t, s)>0$. It is clearly that $g_{2}(t, s)>0$, therefore $G(t, s)>$ 0 , for $t, s \in(0,1)$. 
(2) Since

$$
\begin{aligned}
& \frac{\partial g_{1}(t, s)}{\partial t}=\frac{(\alpha-1)(1-s)^{\alpha-2}-(\alpha-1)(t-s)^{\alpha-2}}{\Gamma(\alpha)}>0 \\
& \frac{\partial g_{2}(t, s)}{\partial t}=\frac{(1-s)^{\alpha-2}}{\Gamma(\alpha-1)}>0 .
\end{aligned}
$$

we know $G(t, s)$ is increasing with respect to $t$. Consequently,

$$
0 \leq G(t, s) \leq \max _{0 \leq t \leq 1} G(t, s)=G(1, s), \text { for } t, s \in[0,1] \text {. }
$$

One has

$$
\begin{aligned}
& \min _{1 / 4 \leq t \leq 3 / 4} G(t, s)=G\left(\frac{1}{4}, s\right)= \begin{cases}\frac{\frac{1}{4}(\alpha-1)(1-s)^{\alpha-2}-\left(\frac{1}{4}-s\right)^{\alpha-1}}{\Gamma(\alpha)}, & s \in\left(0, \frac{1}{4}\right], \\
\frac{\frac{1}{4}(1-s)^{\alpha-2}}{\Gamma(\alpha-1)}, & s \in\left[\frac{1}{4}, 1\right) .\end{cases} \\
& \max _{0 \leq t \leq 1} G(t, s)=G(1, s)=\frac{(\alpha-1)(1-s)^{\alpha-2}-(1-s)^{\alpha-1}}{\Gamma(\alpha)}, s \in(0,1) .
\end{aligned}
$$

we will prove

$$
\min _{1 / 4 \leq t \leq 3 / 4} G(t, s) \geq \frac{1}{4} \max _{0 \leq t \leq 1} G(t, s)=\frac{1}{4} G(1, s)
$$

As follows,

$1^{0}:$ For $0<s \leq \frac{1}{4}$,

$$
\begin{gathered}
\min _{1 / 4 \leq t \leq 3 / 4} G(t, s)=\frac{(\alpha-1)(1-s)^{\alpha-2}}{4 \Gamma(\alpha)}-\frac{\left(\frac{1}{4}-s\right)^{\alpha-1}}{\Gamma(\alpha)} \\
\frac{1}{4} \max _{0 \leq t \leq 1} G(t, s)=\frac{1}{4} G(1, s)=\frac{(\alpha-1)(1-s)^{\alpha-2}}{4 \Gamma(\alpha)}-\frac{(1-s)^{\alpha-1}}{4 \Gamma(\alpha)}
\end{gathered}
$$

Since $2<\alpha \leq 3,0<s \leq \frac{1}{4}$ we can obtain

$$
\left(\frac{1}{4}-s\right)^{\alpha-1}=\left(\frac{1}{4}\right)^{\alpha-1}(1-4 s)^{\alpha-1} \leq \frac{1}{4}(1-4 s)^{\alpha-1}<\frac{1}{4}(1-s)^{\alpha-1}
$$

Thus,

$$
\min _{1 / 4 \leq t \leq 3 / 4} G(t, s) \geq \frac{1}{4} \max _{0 \leq t \leq 1} G(t, s)=\frac{1}{4} G(1, s) .
$$

$2^{0}:$ For $\frac{1}{4} \leq s<1$,

$$
\begin{gathered}
\min _{1 / 4 \leq t \leq 3 / 4} G(t, s)=\frac{\frac{1}{4}(1-s)^{\alpha-2}}{\Gamma(\alpha-1)}=\frac{(\alpha-1)(1-s)^{\alpha-2}}{4 \Gamma(\alpha)} \\
\frac{1}{4} \max _{0 \leq t \leq 1} G(t, s)=\frac{1}{4} G(1, s)=\frac{(\alpha-1)(1-s)^{\alpha-2}}{4 \Gamma(\alpha)}-\frac{(1-s)^{\alpha-1}}{4 \Gamma(\alpha)}
\end{gathered}
$$

It is clearly that

$$
\min _{1 / 4 \leq t \leq 3 / 4} G(t, s) \geq \frac{1}{4} \max _{0 \leq t \leq 1} G(t, s)=\frac{1}{4} G(1, s) .
$$

The proof is complete. 
Lemma 1.7. If $f \in C[0,1]$, and $f \geq 0$, then, the unique solution $u$ of problem (1.1) satisfies

$$
\min _{\frac{1}{4} \leq t \leq \frac{3}{4}} u(t) \geq \frac{1}{4}\|u\|
$$

Proof. By Lemma 1.5, $u$ can be expressed by

$$
u(t)=\int_{0}^{1} G(t, s) f(s, u(s)) d s \leq \int_{0}^{1} \max _{0 \leq t \leq 1} G(t, s) f(s, u(s)) d s
$$

SO,

$$
\max _{0 \leq t \leq 1} \int_{0}^{1} G(t, s) f(s, u(s)) d s \leq \int_{0}^{1} \max _{0 \leq t \leq 1} G(t, s) f(s, u(s)) d s
$$

then,

$$
\|u\|=\max _{0 \leq t \leq 1}|u(t)|=\max _{0 \leq t \leq 1} \int_{0}^{1} G(t, s) f(s, u(s)) d s \leq \int_{0}^{1} \max _{0 \leq t \leq 1} G(t, s) f(s, u(s)) d s
$$

Taking into account(1.4), we obtain

$$
\begin{gathered}
\min _{\frac{1}{4} \leq t \leq \frac{3}{4}} u(t)=\min _{\frac{1}{4} \leq t \leq \frac{3}{4}} \int_{0}^{1} G(t, s) f(s, u(s)) d s \\
\geq \frac{1}{4} \int_{0}^{1} \max _{0 \leq t \leq 1} G(t, s) f(s, u(s)) d s \geq \frac{1}{4} \max _{0 \leq t \leq 1} \int_{0}^{1} G(t, s) f(s, u(s)) d s=\frac{1}{4}\|u\|
\end{gathered}
$$

The proof is complete.

Lemma 1.8. [5] Let $X$ be a Banach space, $P \subseteq X$ a cone, and $\Omega_{1}, \Omega_{2}$ are two bounded open balls of $X$ centered at the origin with $\bar{\Omega}_{1} \subset \Omega_{2}$. Suppose that

$A: P \cap\left(\bar{\Omega}_{2} \backslash \Omega_{1}\right) \rightarrow P$ is a completely continuous operator such that either

(i) $\|A x\| \leq\|x\|, x \in P \cap \partial \Omega_{1}$ and $\|A x\| \geq\|x\|, x \in P \cap \partial \Omega_{2}$, or

(ii) $\|A x\| \geq\|x\|, x \in P \cap \partial \Omega_{1}$ and $\|A x\| \leq\|x\|, x \in P \cap \partial \Omega_{2}$

holds. Then $A$ has a fixed point in $P \cap\left(\bar{\Omega}_{2} \backslash \Omega_{1}\right)$.

\section{MAin RESUlts}

In this section, by uses of Lemma 1.5, Lemma 1.6,Lemma 1.7 and Lemma 1.8, we will obtain the existence of positive solution with semipositone nonlinearity for Problem (1.1).

Let $X=C[0,1]$ be endowed with the ordering $u \leq v$ if $u(t) \leq v(t)$ for all $t \in[0,1]$, and the maximum norm

$$
\|u\|=\max _{0 \leq t \leq 1}|u(t)|
$$

Define the cone $K \subset X$ by

$$
K=\left\{u \in X \mid u(t) \geq 0, \text { and } \min _{\frac{1}{4} \leq t \leq \frac{3}{4}} u(t) \geq \frac{1}{4} \max _{0 \leq t \leq 1}|u(t)|=\frac{1}{4}\|u\|,\right\}
$$


Define operator $T: K \rightarrow K$

$$
T u(t)=\int_{0}^{1} G(t, s) f(s, u(s)) d s
$$

Lemma 2.1. $T: K \rightarrow K$ is completely continuous.

Proof. Let $u \in K$, we have

$$
T u(t)=\int_{0}^{1} G(t, s) f(s, u(s)) d s \leq \int_{0}^{1} \max _{0 \leq t \leq 1} G(t, s) f(s, u(s)) d s
$$

So,

$$
\max _{0 \leq t \leq 1} T u(t)=\max _{0 \leq t \leq 1} \int_{0}^{1} G(t, s) f(s, u(s)) d s \leq \int_{0}^{1} \max _{0 \leq t \leq 1} G(t, s) f(s, u(s)) d s
$$

Taking into account (1.4), we get

$$
\begin{gathered}
\min _{\frac{1}{4} \leq t \leq \frac{3}{4}} T u(t)=\min _{\frac{1}{4} \leq t \leq \frac{3}{4}} \int_{0}^{1} G(t, s) f(s, u(s)) d s \\
\geq \frac{1}{4} \int_{0}^{1} \max _{0 \leq t \leq 1} G(t, s) f(s, u(s)) d s \geq \frac{1}{4} \max _{0 \leq t \leq 1} \int_{0}^{1} G(t, s) f(s, u(s)) d s=\frac{1}{4}\|T u\|
\end{gathered}
$$

Also, by Lemma1.6, for $u \in K$, we have $T u(t) \geq 0,0 \leq t \leq 1$.

Consequently, $T: K \rightarrow K$. Further, with the nonnegativeness and continuity of $G(t, s)$ and $f(t, u)$, by means of Arzela-Ascoli theorem we can easily obtain $T$ is completely continuous. The proof is complete.

Theorem 2.2. Suppose

$\left(H_{1}\right) f \in C([0,1] \times[0, \infty),(-\infty,+\infty)), \exists M>0, L>-M$, such that $-M \leq f(t, x) \leq L, 0 \leq t \leq 1,0 \leq x \leq 1$;

$\left(H_{2}\right) \lim _{x \rightarrow \infty} \frac{f(t, x)}{x}=+\infty, t \in[\alpha, \beta] \subset[0,1]$, uniformly hold.

Then Problem (1.1) has at least one positive solution.

Proof. Let

and set

$$
w(t)=\int_{0}^{1} G(t, s) d s=\frac{\alpha t-t^{\alpha}}{\Gamma(\alpha+1)}<\frac{1}{\Gamma(\alpha)}
$$

$$
v(t)=\frac{1}{4} M w(t)<\frac{\frac{1}{4} M}{\Gamma(\alpha)}, u(t)=\overline{u(t)}+v(t)
$$

then $\overline{u(t)}$ is the positive solution of problem(1.1) if and only if $u(t)$ is the solution of the following problem:

$$
\left\{\begin{array}{l}
D_{0^{+}}^{\alpha} u(t)+[f(t, u(t)-v(t))+M]=0, \quad 0<t<1, \\
u(0)=u^{\prime}(1)=u^{\prime \prime}(0)=0
\end{array}\right.
$$

and satisfies $u(t)-v(t)>0,(0<t<1)$.

Define the operator $T^{*}: K \rightarrow K$

$$
T^{*} x(t)=\int_{0}^{1} G(t, s)[f(s, x(s)-v(s))+M] d s
$$


From Lemma2.1, we know $T^{*}: K \rightarrow K$ is completely continuous operator. Clearly, the fixed point of operator $T^{*}$ is the solution of problem (2.1).

Let

we get

$$
K_{1}=\left\{x \in K:\|X\|<\frac{M+L}{\Gamma(\alpha)}\right\} \text { for } u \in \partial K_{1}
$$

$$
\begin{gathered}
\|T x\|=\max _{0 \leq t \leq 1} \int_{0}^{1} G(t, s)[f(s, x(s)-v(s))+M] d s \\
\leq(M+L) \max _{0 \leq t \leq 1} \int_{0}^{1} G(t, s) d s<\frac{M+L}{\Gamma(\alpha)}=\|x\|
\end{gathered}
$$

Thus, $\|T x\|=\|x\|$, for $u \in \partial K_{1}$.

By condition $\left(H_{2}\right)$ exists $R>0$ is large enough such that $R>\frac{2 M}{\Gamma(\alpha)}$ satisfies

$$
\frac{f(t, x)+M}{x} \geq N, \text { for } t \in[\xi, \eta] \subset[0,1], x \geq \frac{R}{8}
$$

where $N$ is chosen so that

$$
\frac{\frac{1}{4} N}{2} \int_{\xi}^{\eta} G\left(\frac{\xi+\eta}{2}, s\right) d s>1
$$

Set

$$
K_{R}=\{x \in K:\|x\|<R\}, \text { for } x \in \partial K_{R} .
$$

we have

$$
x(t)>\frac{1}{4}\|x\|=\frac{1}{4} R>\frac{M}{2 \Gamma(\alpha)}>2 v(t)
$$

SO

$$
\begin{gathered}
x(t)-v(t)>x(t)-\frac{1}{2} x(t)=\frac{1}{2} u(t)>\frac{R}{8}, t \in[0,1] \\
x(t)-v(t)>\frac{R}{8}, t \in[\xi, \eta]
\end{gathered}
$$

Thus, we obtain

$$
f(t, x(t)-v(t))+M \geq N(x(t)-v(t)) \geq \frac{R}{8}, t \in[\xi, \eta] .
$$

Consequently,

$$
\begin{gathered}
T x(t)=\int_{0}^{1} G\left(\frac{\xi+\eta}{2}, s\right)[f(s, x(s)-v(s))+M] d s \\
\geq \int_{\xi}^{\eta} G\left(\frac{\xi+\eta}{2}, s\right) \frac{N R \frac{1}{4}}{2} d s \geq \frac{N R \frac{1}{4}}{2} \int_{\xi}^{\eta} G\left(\frac{\xi+\eta}{2}, s\right) d s>R=\|x\|
\end{gathered}
$$

Therefore, $\|T x\|=\|x\|$, for $x \in \partial K_{R}$. An application of Lemma1.8 yields that exists $u \in K_{R} \backslash \overline{K_{1}}$, such that $u=T u$.

Namely, $u=u(t)$ satisfies 


$$
\left\{\begin{array}{l}
D_{0^{+}}^{\alpha} u(t)+[f(t, u(t)-v(t))+M]=0, \quad 0<t<1 \\
u(0)=u^{\prime}(1)=u^{\prime \prime}(0)=0
\end{array}\right.
$$

thus,

$$
\begin{gathered}
u(t) \geq \frac{1}{4}\|u\|=\frac{1}{4} \max _{0 \leq t \leq 1}|u(t)| \\
=\frac{1}{4} \max _{0 \leq t \leq 1}\left|\int_{0}^{1} G(t, s)[f(s, x(s)-v(s))+M] d s\right| \\
\geq \frac{1}{4} \cdot M \max _{0 \leq t \leq 1} \int_{0}^{1} G(t, s) d s=\frac{\frac{1}{4} M}{\Gamma(\alpha)}>v(t)
\end{gathered}
$$

Consequently,

$$
\overline{u(t)}=u(t)-v(t)>0,0<t \leq 1 \text {. }
$$

and satisfies

$$
\left\{\begin{array}{l}
D_{0^{+}}^{\alpha} u(t)+f(t, u(t))=0, \quad 0<t<1, \\
u(0)=u^{\prime}(1)=u^{\prime \prime}(0)=0,
\end{array}\right.
$$

Obviously, $\overline{u(t)} 0<t<1$ is positive solution of problem (1.1). The proof is complete.

\section{REFERENCES}

1. Z.B. Bai, Positive solutions for boundary value problem of nonlinear fractional differential equation, J. Math. Anal. Appl. 311 (2005) 495-505.

2. A. Babakhani and V.D. Gejji, Existence of positive solutions of nonlinear fractional differential equations, J. Math. Anal. Appl. 278 (2003) 434-442. 1

3. D. Delbosco and L. Rodino, Existence and uniqueness for a nonlinear fractional differential equation, J. Math. Anal. Appl. 204 (1996) 609-625. 1

4. A.M.A. El-Sayed, Nonlinear functional differential equations of arbitrary orders, Nonlinear Analysis TMA 33 (1998) 181-186. 1

5. V.D. Gejji and A. Babakhani, Analysis of a system of fractional differential equations, $J$. Math. Anal. Appl. 293 (2004) 511-522. 1, 1.8

6. A.A. Kilbas, O.I. Marichev, and S.G. Samko, Fractional Integral and Derivatives (Theory and Applications). Gordon and Breach, Switzerland, 1993. 1, 1.4

7. A.A. Kilbas and J.J. Trujillo, Differential equations of fractional order: methods, results and problems-I, Applicable Analysis 78 (2001) 153-192. 1

8. A.A. Kilbas and J.J. Trujillo, Differential equations of fractional order: methods, results and problems-II, Applicable Analysis 81 (2002) 435-493. 1

9. K.S. Miller, Fractional differential equations, J. Fract. Calc. 3 (1993) 49-57. 1

10. K.S. Miller and B. Ross, An Introduction to the Fractional Calculus and Fractional Differential Equations, Wiley, New York, 1993.

11. S.Q. Zhang, The existence of a positive solution for a nonlinear fractional differential equation, J. Math. Anal. Appl. 252 (2000) 804-812. 1

12. S.Q. Zhang, Existence of positive solution for some class of nonlinear fractional differential equations, J. Math. Anal. Appl. 278 (2003) 136-148. 1 
13. Q.L. Yao, Existence of positive solution for a third order three point boundary value problem with semipositone nonlinearity, Journal of Mathematical Research Exposition. 23 (2003) 591-596.

1 Department of Mathematics, Shandong University of Science and TechnolOGY,QINGDAO, 266510, PRC.

E-mail address: qiutingting19833@163.com

2 Department of Mathematics, Shandong University of Science and TechnolOGY, QIngdaO, 266510, PRC.

E-mail address: zhanbingbai@163.com 